\title{
La résilience dans le parcours scolaire des jeunes noirs d'origine africaine et caribéenne au Québec ${ }^{1}$
}

\author{
Pierre Canisius Kamanzi \\ Université de Montréal
}

\section{Résumé}

Cet article analyse les parcours scolaires d'étudiants québécois noirs d'origine immigrée de l'Afrique subsaharienne et des Caraïbes, ayant en commun de faire partie de groupes racisés, d'être la cible de discrimination et de marginalisation, et d'être à risque de parcours scolaires fragiles. Les résultats obtenus à partir des données longitudinales $(N=8415)$ révèlent cependant que ces élèves sont à même de contourner ces obstacles. Bien qu'ils soient plus à risque de difficultés scolaires majeures, de retards dus au redoublement, moins susceptibles de fréquenter un établissement privé ou d'être admis aux programmes enrichis, ces élèves accèdent aux études postsecondaires (collège et université) dans une proportion plutôt comparable à celle de leurs pairs dont les parents sont de souche eurocanadienne. Bien que la situation soit quelque peu inversée en ce qui a trait à l'obtention du diplôme,

1 L'auteur remercie chaleureusement les évaluateurs de cet articles pour leurs commentaires modérés et constructifs, ainsi que et Louis D. Tonton Tché et Stéphanie Dumont pour la relecture minutieuse du texte.

Canadian Journal of Education / Revue canadienne de l'éducation 44:1 (2021)

(C)2021 Canadian Society for the Study of Education/ Société canadienne pour l'étude de l'éducation 
cet article appuie l'hypothèse de résilience avancée par certains auteurs, comme Krahn et Taylor (2005), à propos de ces étudiants.

Mots-clés : discrimination et marginalisation, élèves noirs, immigration et immigrés, parcours scolaire et postsecondaire, race et racisme, résilience

\begin{abstract}
This article analyzes the educational pathways of Black Québécois students with immigrant background from Sub-Saharan Africa and the Caribbean, all of whom are members of racialized groups, targets of discrimination and marginalization, and are at risk of vulnerable educational pathways. However, the results obtained from longitudinal data $(N=8415)$ show that these students are able to overcome these obstacles. Although they are more at risk of major academic difficulties, delays due to grade repetition, and are less likely to attend a private school or be admitted to enriched programs, these students access postsecondary education (college and university) in a proportion fairly comparable to that of their peers whose parents are Eurocanadian-born. Although the situation is somewhat reversed with respect to graduation, this article supports the resilience hypothesis put forward by some authors, such as Krahn and Taylor (2005), with respect those students. Keywords: discrimination and marginalization, Black students, immigration and immigrants, school and postsecondary pathways, race and racism, resilience
\end{abstract}




\section{Introduction}

Plusieurs études menées au Canada révèlent que, contrairement à plusieurs autres pays industrialisés, les jeunes d'origine immigrée ont des performances scolaires comparables à celles de leurs pairs issus de parents de souche eurocanadienne, c'est-àdire de Canadiens de naissance d'origine européenne (Cheng et Yan, 2018 ; Hochschild et Cropper, 2010 ; Rothon et al., 2009). De plus, les jeunes issus de familles immigrées poursuivent les études postsecondaires dans une proportion relativement plus élevée (Finnie et Mueller, 2008, 2010 ; Kamanzi et al., 2016 ; Picot, 2012 ; Thiessen, 2009). Toutefois, au-delà de ce portrait général, des analyses poussées ont mis en évidence quelques disparités importantes selon les origines ethnoculturelles et géographiques de leurs parents. Si les jeunes d'origine immigrée européenne et de l'Asie orientale ont des parcours exceptionnels par rapport à la moyenne, la situation est un peu inversée chez leurs pairs dont les parents viennent des Philippines, des Caraïbes, de l'Amérique latine et de l'Afrique subsaharienne (Abada et al., 2009 ; Thiessen, 2009).

Au niveau provincial, des constats similaires ont été observés au Québec (Livingstone et Weinfeld, 2017 ; Mc Andrew et al., 2013) et en Ontario (Abada et al., 2009 ; Livingstone et Weinfeld, 2017 ; Robson et al., 2018 ; Sweet et al., 2010). De manière générale, les jeunes d'origine immigrante ont des parcours scolaires assez comparables à ceux de leurs pairs dont les parents sont eurocanadiens, mais il existe aussi d'importantes disparités entre les communautés. D'un côté, les jeunes dont les parents ont immigré des pays européens, asiatiques ou arabes ont des parcours scolaires comparables, voire de meilleure qualité, à ceux de leurs pairs dont les parents sont de souche eurocanadienne. D'un autre côté, ceux dont les parents ont immigré des Caraïbes, de l'Afrique subsaharienne, de l'Amérique centrale et du Sud et de l'Asie du Sud ont des taux de réussite plus bas que la moyenne provinciale. Il s'ensuit des inégalités importantes au niveau de l'accès et de la persévérance aux études postsecondaires (Kamanzi, 2019a ; Kamanzi et Collins, 2018 ; Kamanzi et al., 2018 ; Kamanzi et Murdoch, 2011).

Les explications avancées pour comprendre ces inégalités sont multiples et varient selon les auteurs, mais elles oscillent autour de deux axes principaux : les différences de performances scolaires, la discrimination et la marginalisation. Si certains soutiennent que ces inégalités sont l'effet de difficultés d'adaptation scolaire (Thiessen, 2009), 
d'autres les associent au racisme (Collins et Magnan, 2018 ; Livingstone et Weinfeld, 2017) ou à la marginalisation (Lafortune, 2012). Sans nier l'existence des difficultés d'adaptation scolaire ni des ségrégations envers ces élèves/étudiants, ni même réfuter leurs effets, l'objectif de cet article est de montrer qu'une partie d'entre eux arrivent à contourner le destin et persévèrent jusqu'aux études supérieures, ce qui appuie l'hypothèse de la résilience soutenue par des chercheurs comme Krahn et Taylor (2005), ainsi que Anisef et Kilbride (2004).

L'article est divisé en cinq sections. La première propose un aperçu des recherches antérieures sur la discrimination systémique et les parcours scolaires des groupes ethniques marginalisés. La deuxième définit brièvement le concept de résilience, ses principales dimensions et son application en éducation. La troisième section, portant sur la méthodologie, décrit les données utilisées, les variables étudiées et les stratégies d'analyse mobilisées. Dans la quatrième section, nous présentons et interprétons les résultats. Enfin, la discussion propose quelques pistes d'interprétation de ces résultats. Pour terminer, la conclusion récapitule les principaux constats de l'étude.

\section{Discrimination systémique et parcours scolaires des jeunes d'origine immigrée}

Les études à l'échelle internationale font état des discriminations subies par les élèves et les étudiants d'origine immigrée (Levels et al., 2008 ; Söhn et Özcan, 2006), voire de marginalisation directe dans certains contextes (Felouzis et al., 2005). Le degré d'exclusion varie cependant entre pays, selon les politiques publiques d'immigration (de Graauw et Vermeulen, 2016 ; Hadj Abdou, 2019 ; OCDE/UE, 2018) et les traditions idéologiques nationales en matière d'immigration (Alba et Foner, 2014 ; Silver, 2015). Des recherches précisent par ailleurs qu'à l'intérieur d'un même pays, la situation varie selon l'origine et l'appartenance ethnique des immigrants (Safi, 2008), favorisant ainsi une certaine stratification ethnique (Abada et al., 2009 ; Koopmans, 2013).

Les tenants de la Critical Race Theory (Dei, 2013 ; Delgado Bernal, 2002 ; James, 2009 ; Ladson-Billings, 1998 ; Sleeter, 2012) montrent que, derrière les principes de la démocratie et de la justice sociale pourtant reconnus, se dissimule un racisme systémique qui se matérialise par des pratiques et des coutumes courantes de discrimination socialement et politiquement admises. Ces discriminations envers les 
immigrants prennent des formes variées en fonction des contextes nationaux et locaux. En d'autres mots, elles sont institutionnellement ajustées de manière à être socialement admises, contrairement aux formes flagrantes et directes du racisme comme l'esclavage ou l'apartheid (Solorzano, 1998). Dès lors, elles paraissent souvent banales, acceptables ou tolérables, et ne sont pas remises en question. Dans cette perspective, les pratiques de racisme systémique caché n'apparaissent pas comme une aberration, mais bien au contraire, comme une logique rationnelle imposée par la classe sociale dominante pour organiser la société (Sleeter, 2012). Institutionnalisées, ces pratiques servent d'outils pour justifier le maintien des frontières ethniques, des clôtures sociales, de l'infériorisation et de l'exclusion des groupes racisés à partir de différents critères socialement fabriqués (Sala Pala, 2007). Dans le domaine de l'éducation, elles se matérialisent par des stéréotypes et des préjugés dont les effets cumulatifs favorisent, de génération en génération, la production et la reproduction des inégalités de réussite et d'orientation scolaire et professionnelle aux différents paliers du système éducatif (Rogers-Sirin et al., 2014).

Bien que les politiques publiques canadiennes aient toujours promu le multiculturalisme et la tolérance (Bloemraad, 2012 ; Ghosh, 2004 ; Harles, 1997, 2004) et qu'elles investissent beaucoup de ressources pour renforcer l'intégration et l'inclusion sociales des immigrants (Simich et al., 2005), des situations d'exclusion et de discrimination sous-estimées persistent sur le plan institutionnel (Livingstone et Weinfeld, 2017). Celles-ci touchent les membres des communautés dites « racisées », c'est-à-dire exposées au racisme au terme d'un processus de construction sociale qui les assimile à des groupes raciaux tout en niant l'individualité des membres au sein de dynamiques de rapports de pouvoir (Henry et Tator, 2009 ; Henry et al., 2010 ; Mickelson, 2003). C'est ce qu'illustrent, par exemple, Henry et al. (2010) dans leur ouvrage The colour of democracy. Les auteurs mettent en évidence l'existence des attitudes racistes cachées dans l'organisation et les pratiques des institutions canadiennes, dont le gouvernement, la justice, le marché de l'emploi, les médias et l'éducation.

En milieu scolaire, les pratiques de marginalisation et d'exclusion affectent particulièrement les élèves de familles de race noire immigrées des Caraïbes et de l'Afrique subsaharienne, et fragilisent leurs parcours scolaires (Collins et Magnan, 2018 ; Lafortune, 2012 ; Potvin et Leclercq, 2014). Les conditions de vie précaires de leurs familles ne font qu'accentuer les effets de ces pratiques, dès lors que leurs parents sont 
eux-mêmes souvent victimes de la discrimination sur le marché de l'emploi (Briggs, 2019 ; Kamanzi, 2012). Des études soulignent, notamment en milieu urbain, l'existence de pratiques de regroupements à caractère ethnoculturel fondés sur la sélection scolaire, mais aboutissant à une discrimination systémique à l'endroit de certains groupes ethniques racisés par le biais de 1'orientation scolaire (Henry et Tator, 2009 ; Henry et al., 2010 ; James et Turner, 2017 ; Mickelson, 2003). Ces élèves seraient victimes d'un traitement injuste par des pratiques affichées ou cachées fragilisant leurs parcours scolaires à court ou à moyen terme, notamment : l'accès inégal à l'information essentielle et à d'autres ressources pour planifier et structurer un parcours scolaire ambitieux, mais approprié, et l'exclusion par des pratiques de déclassement par les personnes responsables de l'orientation scolaire et professionnelle (Magnan et al., 2019). De telles pratiques favorisent particulièrement la marginalisation des élèves issus des communautés noires. Au Québec, Potvin et Leclercq (2014) ont relevé des pratiques institutionnelles courantes qui consistent à reléguer ces élèves du secteur des jeunes (formation générale) vers celui des adultes (formation professionnelle courte), les écartant ainsi de la possibilité de poursuivre des études postsecondaires.

Comme mentionné au début, la présente étude s'intéresse à la situation spécifique des jeunes Canadiens de la province du Québec issus de parents noirs immigrés d'origine africaine et caribéenne, les plus à risque de mener des parcours scolaires fragiles ou d'échec scolaire. Elle vise à montrer que, malgré les multiples obstacles sociaux et scolaires auxquels ils sont confrontés, une partie d'entre eux persévèrent jusqu'aux études postsecondaires et y obtiennent un diplôme, faisant ainsi preuve de résilience.

\section{Concept de résilience en éducation}

Emprunté au domaine de la physique, puis appliqué à celui des sciences sociales, notamment la psychologie, la sociologie et l'économie, le concept de résilience a plusieurs acceptions, mais celles-ci ont une dimension commune : quelle que soit la discipline à laquelle il est appliqué, ce concept réfère à la capacité et à l'habileté à revenir à l'état stable ou à l'équilibre à la suite d'une perturbation (Bhamra et al., 2011). Chez l'être humain, cette perturbation peut être l'effet d'un évènement temporaire, comme les crises et les catastrophes, ou d'une situation permanente ou de longue durée, comme 
la discrimination, empêchant un individu ou un groupe d'individus d'évoluer selon le rythme normal. La résilience sous-entend alors que les personnes concernées sont dans une situation de vulnérabilité à laquelle elles tentent de survivre ou d'échapper. Autrement dit, ces personnes sont à risque ou exposées à un danger ou à un échec. Deux critères doivent être remplis pour qu'une personne résiliente soit considérée comme telle (Estrada et al., 2016). Premièrement, elle doit faire face à une adversité d'une certaine ampleur (fréquence, intensité et durée) et qui la rend vulnérable. Deuxièmement, malgré cette vulnérabilité, elle doit réussir à atteindre les mêmes objectifs que les autres en situation normale ou régulière, c'est-à-dire non exposée à un danger majeur ou à une source de vulnérabilité. La résilience réfère ainsi à la capacité et à l'habileté de résister devant l'adversité et à « s'en sortir » malgré les obstacles importants (Luthans et al., 2006).

Les facteurs à l'origine de l'adversité et de la vulnérabilité, aussi appelés « facteurs de risque », sont multiples (Anaut, 2006 ; Manciaux, 2001). Ils peuvent être associés aux caractéristiques de l'individu, à son environnement, ou à une combinaison des deux. Dans le cas des caractéristiques individuelles, ils sont innés ou acquis à la suite d'évènements, et ont trait, par exemple, à un handicap ou à une difficulté d'ordre physique ou psychologique. Quant aux facteurs liés à l'environnement, ils renvoient aux contraintes et obstacles imposés par le milieu social et géographique : par exemple, la discrimination, la ségrégation ou toute autre forme de marginalisation sociale, la pauvreté, l'insécurité et l'insalubrité, l'éloignement physique par rapport aux services publics.

La résilience implique aussi ce que les auteurs qualifient de « facteurs de protection » (Anaut, 2006 ; Manciaux, 2001), c'est-à-dire un ensemble de mécanismes personnels permettant de réagir adéquatement à la perturbation, afin de modérer, voire neutraliser ses effets (Gallopin, 2006). Elle peut ainsi être définie comme le processus par lequel l'individu combine ses compétences et ses habiletés afin d'éviter ou maîtriser des situations menaçantes, s'adapter et éviter des échecs (Bhamra et al., 2011). Cette dernière définition permet de distinguer trois composantes de la résilience : le contexte, les ressources mobilisées et les résultats (Estrada et al., 2016). Le contexte renvoie à la situation d'adversité et de vulnérabilité vécue par l'individu à la suite d'évènements ou de situations de défi. Les ressources mobilisées ou les facteurs de protection renvoient, quant à eux, aux habiletés individuelles associées aux caractéristiques par lesquelles une 
personne parvient à résister et à échapper aux effets de cette adversité : par exemple, la flexibilité, le sentiment de confiance, la motivation, l'optimisme, l'engagement, la persévérance et l'adaptation. Enfin, les résultats réfèrent à la réussite, c'est-à-dire l'atteinte des objectifs ciblés.

En éducation, la résilience réfère aux élèves et étudiants qui persévèrent et réussissent leur scolarité malgré les conditions difficiles associées à leurs caractéristiques personnelles ou à celles de l'environnement social et scolaire dans lequel ils évoluent (Anaut, 2006). Les facteurs de risque peuvent être des difficultés personnelles d'adaptation sociale et d'apprentissage scolaire causées par un évènement ou une condition organique personnelle. Il peut s'agir aussi des obstacles liés au contexte social : la précarité économique et la pauvreté de la famille, l'insalubrité du logement, la violence, l'insuffisance ou le déficit du soutien parental. Dans d'autres cas, les facteurs de risque peuvent être associés à l'environnement scolaire : par exemple, la discrimination et la ségrégation par les pairs ou les enseignants, ou l'éloignement géographique de l'école par rapport au lieu de résidence. Quelle que soit la nature des facteurs de risque, l'élève/ étudiant résilient se distingue par sa capacité à mobiliser et à combiner différents facteurs de protection pour réussir ses études. Les facteurs de protection peuvent être individuels (motivation, sentiment de compétence, persévérance, etc.) ou environnementaux (ressources matérielles, soutien psychologique et scolaire, etc.).

Dans la perspective sociologique, cette mobilisation des facteurs de protection pourrait être interprétée comme un choix rationnel ou une stratégie (Boudon, 1973), dans la mesure où elle est principalement fondée sur les décisions individuelles, la volonté et l'intérêt à réussir (Pettit, 2007). Autrement dit, elle peut être considérée comme le résultat d'une analyse coût/bénéfice de la situation au cours de laquelle l'élève ou l'étudiant résilient effectue un choix, celui de s'engager et d'investir davantage de ressources pour réussir. Ce choix implique non seulement des coûts en termes de sacrifices et de ressources supplémentaires, par exemple le temps et l'effort, mais aussi un processus de négociation constante avec l'environnement pour « survivre » à la compétition :

« À chaque instant, la résilience résulte de l'interaction entre l'individu luimême et son entourage, entre les empreintes de sa vie antérieure et le contexte du moment en matière politique, économique, sociale, humaine. Elle résulte aussi de 
l'interaction entre facteurs de risque et facteurs de protection. » (Manciaux, 2001, p. 326)

Pour réussir, l'individu est conscient qu'il doit s'engager dans la compétition et se positionner (Walker et al., 2006).

Cette situation est observée chez les jeunes Canadiens d'origine immigrée : conscients des multiples contraintes auxquelles ils sont soumis, ces derniers s'investiraient davantage à construire des stratégies pour mieux « s'en sortir » (Magnan, Pilote, Grenier et Darchinian, 2017 ; Motti-Stefanidi, 2018 ; Motti-Stefanidi et Masten, 2017). En particulier, les jeunes issus des communautés noires seraient conscients qu'ils doivent travailler plus que les autres pour faire reconnaître leurs compétences (McGee, 2020 ; McGee et al., 2019). La section suivante décrit la source des données utilisées, les variables étudiées et le modèle d'analyse statistique appliqué pour illustrer cette résilience.

\section{Méthodologie}

\section{Source des données et échantillon}

Les données utilisées dans cet article nous ont été fournies par le ministère de l'Éducation et de 1'Enseignement supérieur du Québec à partir du fichier des données administratives. L'échantillon initial était constitué de 24084 élèves/étudiants qui représentent $25 \%$ de la cohorte entrée en 1re année du secondaire en 2002-2003 et observée sur une période de 10 ans, c'est-à-dire jusqu'en 2012-2013. Selon le lieu de résidence, l'analyse montre qu'environ $95 \%$ des élèves d'origine immigrée faisant partie de cet échantillon résidaient dans la ville de Montréal et ses banlieues. Comme l'étude vise à comparer ces élèves à leurs pairs non immigrants, l'analyse se limitera au sous-échantillon d'élèves/ étudiants dont les parents résidaient dans cette région afin d'éviter le biais du contexte géographique et socioéconomique. Le sous-échantillon retenu est composé de 8415 élèves/étudiants, soit 574 dont les parents ont immigré des Caraïbes ou de l'Afrique subsaharienne et 7841 dont les parents sont de souche eurocanadienne. 
La base de données contient en outre quelques renseignements relatifs aux caractéristiques sociodémographiques (genre, pays d'origine des parents) et scolaires (types d'établissement fréquenté à chaque palier d'orientation, présence ou non de retard scolaire) permettant d'analyser les parcours scolaires de ces élèves. Toutefois, comme il s'agit de données administratives et non d'une enquête, ces données sont limitées sur le plan méthodologique. Ainsi, la base ne contient pas d'information sur certains aspects importants caractérisant les parcours des élèves et des étudiants : par exemple, les expériences scolaires telles que perçues ou vécues par les élèves, les aspirations scolaires et professionnelles, ou encore les rapports entre divers acteurs scolaires (les pairs, le personnel des établissements scolaires, les parents et autres). En raison de la confidentialité, la base de données ne contient pas non plus de renseignement sur les résultats scolaires de l'élève. Pour tenter de combler cette lacune, nous avons complété la base de données par certaines informations disponibles dans les recensements Canada de 2006, permettant de caractériser l'environnement socioéconomique dans lequel a évolué l'élève/étudiant. Dans le cadre de cet article, il s'agit du revenu médian des ménages du quartier de résidence.

\section{Mesure des variables étudiées}

Comme nous le verrons dans la section suivante, la présente étude s'appuie sur des analyses de modélisation par équations structurelles. Celle-ci est composée d'équations simultanées constituées de trois catégories de variables : 1) les variables dépendantes, 2) les variables médiatrices et 3) les variables modératrices.

Trois variables dépendantes en lien avec l'inégalité dans l'enseignement postsecondaire sont analysées : 1) l'accès au collège (cégep), 2) l'obtention du diplôme d'études collégiales et 3) l'accès à l'université. Précisons que, dans l'ensemble des provinces canadiennes, l'enseignement postsecondaire est constitué de deux ordres : le collège et l'université. Signalons toutefois qu'au Québec, il s'agit de deux paliers successifs et non parallèles. Autrement dit, l'admission à l'université est conditionnelle à la détention d'un diplôme d'études collégiales, ce qui n'est pas le cas des autres provinces et territoires canadiens où les deux paliers d'enseignement sont plutôt parallèles.

En ce qui concerne les variables médiatrices, l'analyse tient compte : 1) de l'origine socioéconomique de l'élève, 2) du type d'établissement fréquenté et 3) 
de la présence ou non de difficultés scolaires majeures, ainsi que des situations de redoublement dans le dossier de l'élève. L'origine socioéconomique a été mesurée par le pourcentage de ménages du quartier de résidence des parents ayant un revenu annuel supérieur à la médiane de la région. Celui-ci permet d'estimer le capital économique caractérisant l'environnement dans lequel a évolué l'élève. Soulignons d'entrée de jeu qu'il s'agit d'une mesure indirecte et approximative de l'origine socioéconomique, qui est de nature écologique, et qu'elle permet seulement d'estimer la probabilité que l'élève ait des parents ayant un revenu élevé. Cette variable a été créée à partir des données tirées du recensement canadien de 2006. En ce qui concerne le type d'école secondaire, les sujets ont été répartis en trois catégories : 1) ceux qui ont fréquenté une école publique, mais ont suivi exclusivement des programmes réguliers ; 2) ceux qui ont fréquenté une école publique, mais ont suivi des cours enrichis (par exemple, programme d'éducation internationale, sciences, arts, sport-études) ; et 3) ceux qui ont fréquenté une école privée. Quant aux difficultés et au redoublement, le dossier de l'élève/étudiant indique s'il a connu de tels épisodes au cours de sa scolarité primaire et secondaire ; la variable est donc dichotomique : 1) oui ou 2) non. Enfin, la variable modératrice dans le cadre de cette étude est l'origine immigrante des parents : 1) Canada (catégorie de référence) ou 2) Afrique subsaharienne ou Caraïbes.

En guise de variables de contrôle, le modèle tient compte des trois caractéristiques sociales suivantes dont l'influence sur les parcours scolaires est traditionnellement reconnue à travers les études antérieures menées au Québec et ailleurs : 1) la langue maternelle et 2) le genre (femme : catégorie de référence). En ce qui concerne la langue maternelle, les sujets ont été regroupés selon l'usage à la maison en trois catégories : 1) francophones (catégorie de référence), 2) anglophones et 3) allophones. Ces variables sont sélectionnées en fonction des données disponibles dans la base de données utilisée.

\section{Modèle d'analyse}

La présente étude s'appuie sur une analyse de modélisation par équations structurales (Byrne, 2010 ; Hoyle, 2012 ; Kline, 2011). Elle examine à la fois l'influence directe et indirecte de l'origine immigrante (caribéenne/subsaharienne) sur l'accès et la persévérance aux études postsecondaires. En ce qui a trait à l'influence directe, comme l'illustrent les figures 1, 2 et 3, il s'agit de l'effet direct de la variable indépendante 
(origine immigrante) sur chacune des trois variables dépendantes (l'accès au collège, à l'université et l'obtention du diplôme). En revanche, l'influence indirecte réfère à l'effet de l'origine immigrante qui s'opère via les variables médiatrices ou intermédiaires. Plus précisément, nous examinons dans quelle mesure l'origine immigrée des parents influe sur le lieu de résidence, lequel influe sur les caractéristiques du parcours au primaire et au secondaire (le type d'établissement secondaire ou de filière fréquenté, les difficultés scolaires majeures et le redoublement), lesquelles influent à leur tour sur les chances d'accès aux études postsecondaires et d'obtention du diplôme. Il s'agit donc d'une chaîne d'influences successives et cumulatives. Pour ce faire, trois analyses de modélisation par équations structurelles seront effectuées sur chacune des trois variables dépendantes mentionnées plus haut : 1) l'accès au collège, 2) l'obtention d'un diplôme d'études collégiales et 3) l'accès à l'université. Chaque analyse est constituée des équations simultanées associées à chacune des variables médiatrices et dépendantes. La première analyse portera sur l'ensemble de l'échantillon $(N=8415)$, alors que la deuxième et la troisième seront effectuées seulement sur les sujets ayant accédé au collège $(n=5425)$.

\section{Présentation des résultats}

\section{Accès au collège après les études secondaires}

Après les études secondaires, les élèves noirs issus des parents immigrés d'origine africaine et caribéenne accèdent au collège dans une proportion (61\%) légèrement inférieure, mais plus ou moins comparable, à celle de leurs pairs dont les parents sont de souche eurocanadienne (65\%) (tableau 1). Bien que le test du Khi carré $\left[\chi^{2}(1)=3.968\right.$, $p=.046]$ révèle que la différence est statistiquement significative au seuil de .05 , celle-ci est relativement faible. 


\section{Tableau 1}

Accès au collège

\begin{tabular}{lccc}
\hline \multicolumn{1}{c}{ Origine } & Non & Oui & $n$ \\
\hline Afrique/Caraïbes & $39 \%$ & $61 \%$ & 574 \\
Canada & $35 \%$ & $65 \%$ & 7841 \\
\hline
\end{tabular}

Les analyses de modélisation par équations structurelles qui suivent examinent si ces constats se maintiennent ou changent lorsqu'on tient compte des variables socioscolaires caractérisant les parcours scolaires des deux groupes d'étudiants. La première analyse porte sur l'accès au collège et la seconde à l'université. Dans les deux cas, nous avons appliqué une analyse de régression logistique binaire par la fonction logit (Allison, 2012).

Les résultats (figure 1) mettent en évidence l'influence directe et indirecte inversée de l'origine immigrante de l'étudiant sur l'accès au collège. En ce qui concerne l'influence directe, ils révèlent une différence statistiquement significative en faveur des étudiants issus de parents noirs immigrés de l'Afrique subsaharienne et des Caraïbes, qui seraient plus susceptibles d'accéder au collège $(\gamma=.67, p<.001)$. Ce résultat apparaît cependant contre-intuitif, dès lors qu'ils sont plus prédisposés à des parcours scolaires qui contribueraient plutôt à les éloigner des études postsecondaires. Ils ont nettement moins de chance de fréquenter une école privée $(\gamma=.50, p<.001)$ ou d'être admis dans des programmes enrichis au public $(\gamma=.73, p<.001)$ que leurs pairs des familles de souche eurocanadienne. En revanche, ils sont davantage enclins à connaître des épisodes de difficultés scolaires ou de retards (redoublement) au primaire comme au secondaire $(\gamma=$ $1.03, p<.001)$. Cela s'expliquerait en partie par le fait que leurs parents ont des revenus faibles et sont plus susceptibles d'habiter dans des quartiers défavorisés $(\gamma=.96, p<$ .001). Or, comme le révèle notre analyse, la probabilité d'accéder aux études collégiales varie sensiblement selon le type d'établissement secondaire fréquenté, la qualité de la scolarité au secondaire et l'origine socioéconomique. Elle est nettement moins élevée chez les élèves ayant suivi des programmes exclusivement réguliers dans une école publique (catégorie de référence), en comparaison aux élèves ayant fréquenté une école privée $(\gamma=1.90, p<.001)$ ou publique, mais ayant suivi des programmes enrichis dans une partie ou la totalité des matières $(\gamma=1.48, p<.001)$. À l'inverse, cette probabilité tend à diminuer sensiblement chez ceux ayant connu des épisodes de difficultés majeures 
et de retards (redoublement) scolaires $(\gamma=1.94, p<.001$ ), ainsi que chez ceux dont les parents résident dans un quartier défavorisé, et vice versa $(\gamma=.28, p<.001)$.

Le fait que les élèves ayant des parents natifs d'Afrique subsaharienne ou des Caraïbes soient proportionnellement plus nombreux à remplir cette triple condition, comme illustré précédemment, réduit forcément leur probabilité d'accéder au collège et, plus largement, aux études postsecondaires. Dès lors, leur taux élevé d'accès au collège appuie l'hypothèse de résilience.

Enfin, concernant le genre et la langue maternelle, la probabilité d'accès au collège est plus élevée chez les anglophones $(\gamma=.56, p<.001)$ et les allophones $(\gamma=.66$, $p<.001$ ), en comparaison aux francophones (catégorie de référence). Elle est également moins élevée pour les hommes $(\gamma=.78, p<.001)$ que pour les femmes.

\section{Figure 1}

Modélisation par équations structurelles - accès au collège

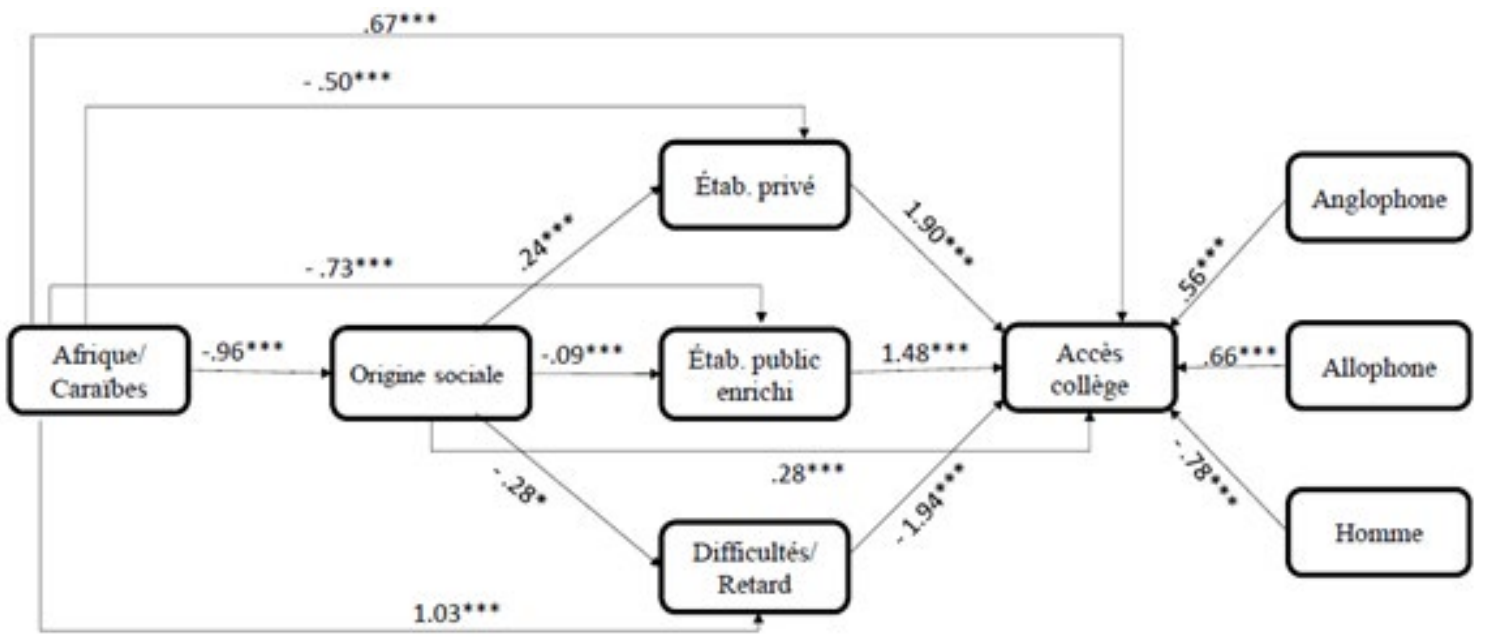

Note. ${ }^{*} p<.05 ; * * * p<.001$

\section{Persévérance et obtention du diplôme}

Accéder aux études postsecondaires est une chose, obtenir un diplôme en est cependant une autre, comme l'indique l'analyse suivante (tableau 2). Dix ans après l'entrée en 1re année du secondaire, $62 \%$ des élèves dont les parents sont de souche eurocanadienne 
avaient obtenu un diplôme d'études collégiales. Ce taux est toutefois nettement moins élevé chez leurs pairs d'origine africaine et caribéenne (40\%). Le test du Khi carré $\left[\chi^{2}(2)\right.$ $=69.10, p=<.001]$ révèle que la différence est statistiquement significative.

\section{Tableau 2}

Obtention du diplôme d'études collégiales

\begin{tabular}{lccc}
\hline \multicolumn{1}{c}{ Origine } & Non & Oui & $n$ \\
\hline Afrique/Caraïbes & $60 \%$ & $40 \%$ & 348 \\
Canada & $38 \%$ & $62 \%$ & 5077 \\
\hline
\end{tabular}

L'analyse de modélisation par équations structurelles (figure 2) montre que cette différence demeure très élevée lorsqu'on tient compte des variables scolaires et sociodémographiques caractérisant le parcours au primaire et au secondaire. La variable étant dichotomique, nous appliquons également l'analyse de régression logistique binaire avec la fonction logit (Allison, 2012). L'analyse montre que les étudiants noirs d'origine africaine et caribéenne inscrits au collège sont moins susceptibles d'avoir obtenu leur diplôme à l'âge de 22 ans $(\gamma=.50, p<.001)$.

Par-delà l'influence directe, leur appartenance ethnique exerce une influence indirecte. Comme l'illustrent les résultats, avoir fréquenté un établissement privé ( $\gamma=.95$, $p<.001)$ ou suivi des programmes enrichis dans un établissement public $(\gamma=.79, p<$ $.001)$ augmente sensiblement les chances d'avoir obtenu un diplôme d'études collégiales à l'âge de 22 ans. À l'inverse, ces chances se trouvent réduites chez les étudiants ayant connu des épisodes de difficultés scolaires ou de redoublement $(\gamma=1.22, p<.001)$. 
CAPSUlE SPÉCIALE : LA RÉUSSITE ÉDUCATIVE DES AFRO-CANADIENS

\section{Figure 2}

Modélisation par équations structurelles - diplôme d'études collégiales

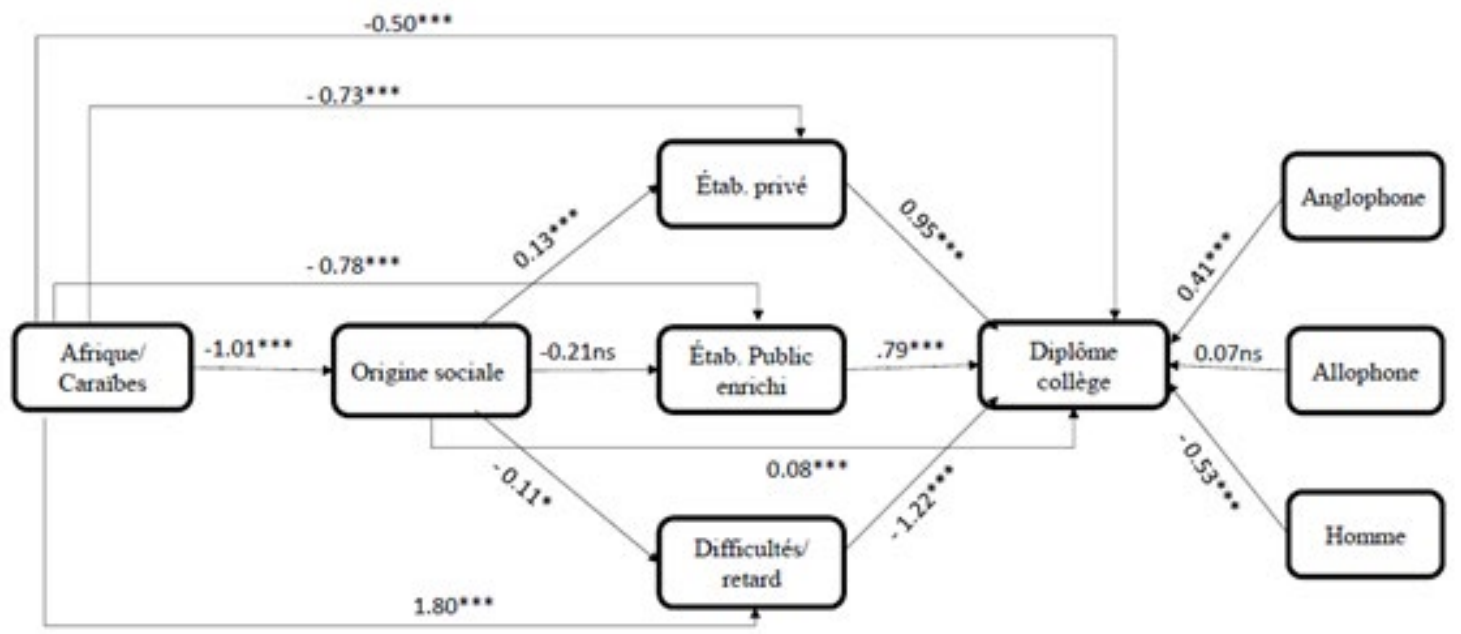

Note. ${ }^{*} p<.05 ; * * * p<.001 ;$ ns : non significatif au seuil de .05

\section{Passage du collège à l'université}

Contrairement au collège, l'université s'avère moins accessible aux jeunes d'origine africaine et caribéenne (tableau 3). Parmi les étudiants ayant fréquenté un collège, le taux de passage à l'université est de $53 \%$ chez les étudiants dont les parents sont de souche eurocanadienne, soit 1.5 fois plus élevé par rapport à leurs pairs dont les parents sont d'origine africaine et caribéenne (36\%). Le test du Khi carré $\left[\chi^{2}(1)=35.98, p<.001\right]$ révèle que la différence est statistiquement significative.

\section{Tableau 3}

Accès à l'université

\begin{tabular}{|l|c|c|c|}
\hline \multicolumn{1}{|c|}{ Origine } & Non & Oui & $n$ \\
\hline Afrique/Caraïbes & $64 \%$ & $36 \%$ & 348 \\
\hline Canada & $47 \%$ & $53 \%$ & 5077 \\
\hline
\end{tabular}


L'analyse de modélisation par équations structurelles (figure 3) révèle que cette différence se maintient même lorsqu'on tient compte des caractéristiques socioéconomiques du quartier de résidence des parents et des deux variables caractérisant le parcours au primaire et au secondaire (type d'établissement fréquenté et la présence de difficultés scolaires majeures). Comme pour les analyses précédentes, cette différence serait l'effet combiné des influences directe et indirecte de l'appartenance ethnique des étudiants noirs. En plus de son influence négative directe $(\gamma=.34, p<.001)$, celle-ci exerce également une influence négative indirecte par l'intermédiaire des trois variables médiatrices : l'origine sociale $(\gamma=1.01, p<.001)$, la fréquentation d'une école secondaire privée $(\gamma=.73, p$ $<.001)$ ou publique enrichie $(\gamma=.73, p<.001)$ et le fait d'avoir connu des difficultés scolaires $(\gamma=1.80, p<.001)$. Or, les résultats montrent que ces variables exercent une influence significative sur l'accès à l'université. Les résultats s'avèrent d'ailleurs similaires à ceux observés précédemment à propos de l'accès au collège et l'obtention du diplôme, bien que l'ampleur de cette influence diminue légèrement selon : la fréquentation d'un établissement privé $(\gamma=1.19, p<.001)$ ou public avec des programmes enrichis $(\gamma=.87, p$ $<.001)$, ou le fait d'avoir connu des difficultés scolaires au secondaire $(\gamma=1.10, p<.001)$.

\section{Figure 3}

\section{Modélisation par équations structurelles - accès à l'université}

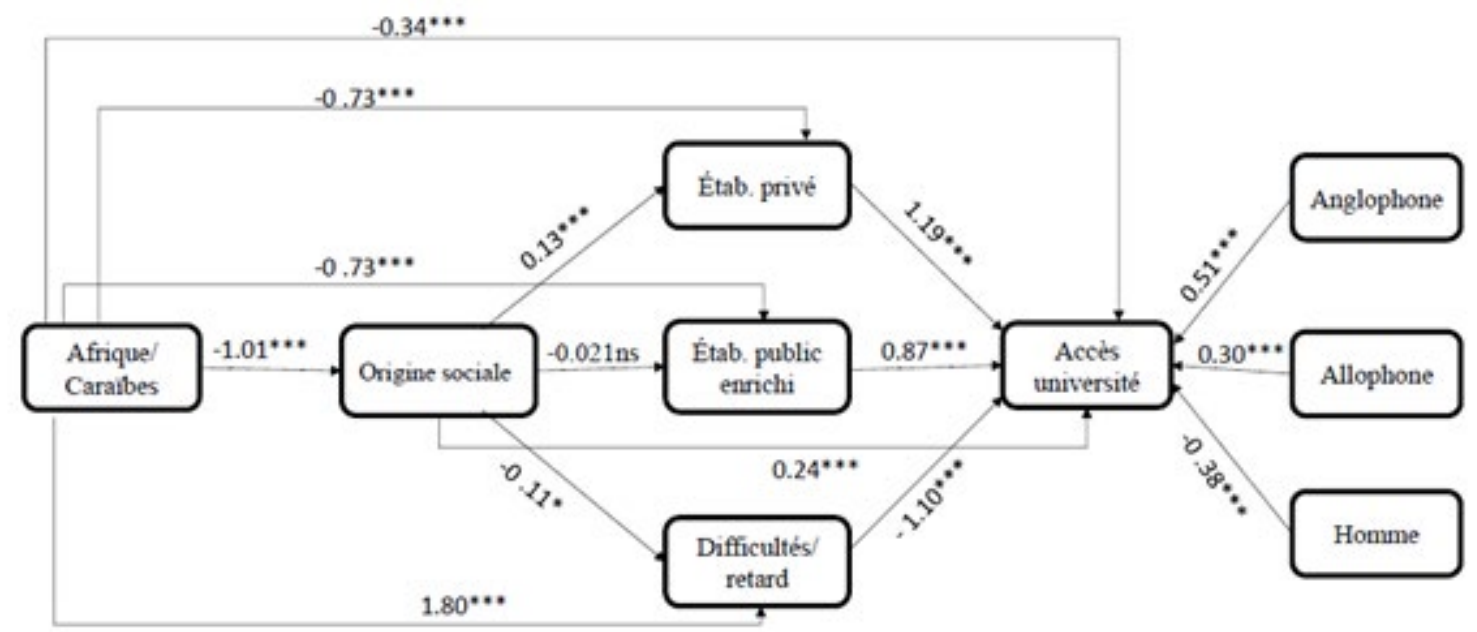

Note. ${ }^{*} p<.05 ; * * * p<.001 ;$ ns : non significatif au seuil de .05 
Il aurait été intéressant d'examiner l'obtention du diplôme d'université, mais les effectifs se sont avérés trop faibles pour effectuer cette analyse. Nous sommes cependant persuadé que, si la taille du sous-échantillon était suffisante, les résultats seraient similaires.

\section{Discussion}

L'objectif de cet article était d'analyser les parcours scolaires d'étudiants québécois noirs d'origine immigrée de l'Afrique subsaharienne et des Caraïbes ayant en commun de faire partie des groupes racisés, d'être la cible de racisme et de discriminations sociales et scolaires, et d'être à risque de parcours scolaires fragiles. Les résultats obtenus à partir des données longitudinales révèlent cependant qu'une partie de ces élèves arrivent à surmonter ces obstacles. Bien qu'ils soient plus à risque de difficultés scolaires majeures et de retards dus au redoublement, et donc moins susceptibles de fréquenter un établissement privé ou d'être admis aux programmes enrichis, ces élèves accèdent au collège dans une proportion plus ou moins comparable à celle de leurs pairs dont les parents sont de souche eurocanadienne.

Ces résultats appuient l'hypothèse de résilience avancée par certains auteurs, comme Krahn et Taylor (2005) ainsi que Anisef et Kilbride (2004), à propos des étudiants issus de parents d'origine caribéenne. Cette hypothèse doit cependant être nuancée, car la situation est quelque peu inversée en ce qui a trait à l'accès à l'université et à l'obtention du diplôme d'études postsecondaires (collégiales ou universitaires). En d'autres mots, la résilience connaît des limites : dix ans après l'entrée en 1re année du secondaire, les élèves dont les parents sont de souche eurocanadienne sont 1.5 fois plus susceptibles d'avoir obtenu un diplôme d'études collégiales (1.55) et d'avoir accédé aux études universitaires (1.47). Des analyses de modélisations par équations structurelles révèlent que ces différences se maintiennent même lorsqu'on tient compte des variables socioscolaires associées au parcours antérieur (primaire et secondaire).

Deux pistes explicatives pourraient être avancées pour comprendre ces différences. La première est que nombre de ces étudiants ont des parcours caractérisés par des épisodes de redoublement au primaire et au secondaire et arrivent aux études postsecondaires avec un certain retard d'une année ou deux, comme en témoignent 
les travaux de Kamanzi et al. (2016) à l'échelle canadienne. La deuxième est qu'ils sont plus susceptibles d'avoir connu des difficultés scolaires majeures au secondaire. Dès lors, ils seraient plus susceptibles de suivre des cours de rattrapage ou de mise à niveau avant d'être admis officiellement aux études collégiales. Une étude récente de Kamanzi et al. (2018) montre que ces étudiants sont plus susceptibles d'emprunter des parcours non linéaires au secondaire, combinant la formation générale et professionnelle avant d'entreprendre les études postsecondaires au niveau collégial. Comme souligné précédemment, ces bifurcations de parcours résulteraient, en partie, des pratiques de déclassement par les services d'orientation et les enseignants (Magnan et al., 2019 ; Potvin et Leclercq, 2014). Ces derniers ont tendance à avoir des attentes scolaires nivelées vers le bas à l'endroit de ces élèves, les poussant à opter pour des formations professionnelles courtes. Toutefois, grâce à la flexibilité du système scolaire québécois, il leur est toujours possible de se réorienter vers la formation générale pour répondre aux exigences d'admission au collège (Kamanzi et al., 2017). En raison des allers-retours entre les deux formations, l'accès tardif aux études collégiales serait un facteur de retard dans l'obtention du diplôme et l'accès à l'université.

Par ailleurs, ces étudiants seraient davantage dans l'obligation de concilier les études et le travail salarié pour subvenir à leurs besoins et soutenir leur famille souvent vulnérable sur le plan financier (Briggs, 2019). Les analyses présentées montrent que leurs familles sont nettement plus susceptibles de résider dans des quartiers où le revenu moyen par ménage est inférieur à la médiane régionale. En d'autres mots, ils sont davantage exposés aux conditions de vie précaires. À cela s'ajouteraient, compte tenu de leurs parcours scolaires fragiles et de leurs faibles chances de fréquenter un établissement privé ou d'être admis à des programmes enrichis, leurs performances scolaires souvent moins élevées à la fin des études secondaires. De plus, même lorsqu'ils sont admis aux études postsecondaires, rien n'indique qu'ils soient à l'abri du racisme ; au contraire, des études menées au Québec et ailleurs au Canada témoignent de la persistance de stéréotypes et de préjugés à caractère racial dans les établissements collégiaux et universitaires (Bhopal, 2017 ; Collins et Magnan, 2018 ; Henry et Tator, 2009). Dès lors, le fait qu'ils soient moins susceptibles, à l'âge de 22 ans, d'avoir obtenu un diplôme d'études postsecondaires et d'avoir accédé à l'université ne remet pas en question l'hypothèse de résilience, mais souligne qu'elle est à nuancer. Autrement dit, cette résilience connaît elle-même des limites et n'est pas toujours suffisante pour réussir. 
Cela dit, comment expliquer cette résilience face à l'adversité liée à de multiples facteurs de risque associés à l'environnement social et scolaire que nous venons de décrire ? En d'autres mots, quels seraient leurs facteurs de protection (Anaut, 2006 ; Manciaux, 2001) ? Ces derniers seraient d'ordres individuel et environnemental, et trois pistes explicatives peuvent être avancées. La première a trait au capital culturel des parents. Comme ailleurs au Canada, la plupart des immigrants résidant au Québec détiennent un diplôme d'études postsecondaires et sont plus scolarisés que la moyenne provinciale (Institut de la Statistique du Québec, 2019). À défaut d'autres ressources, notamment le capital social et économique, ils considèrent les études postsecondaires pratiquement comme le seul moyen d'accéder à un emploi de qualité et de réussir l'intégration socioéconomique. Comme peu de familles immigrées parviennent à acquérir le statut socioéconomique souhaité, une scolarisation poussée de leurs enfants constituerait le principal moyen d'envisager la mobilité sociale (Ichou, 2014). En quelque sorte, l'accès à l'enseignement postsecondaire et à des diplômes représenterait une stratégie indirecte de recouvrement. Les parents immigrants chercheraient alors à optimiser le capital culturel dont ils disposent pour aménager un environnement favorable à la persévérance scolaire de leurs enfants (Potochnick, 2014).

La deuxième piste réfère à la mobilisation familiale autour du projet scolaire des enfants (Kanouté et Lafortune, 2010 ; Magnan, Pilote, Grenier et Kamanzi, 2017). Des recherches montrent que les aspirations aux études postsecondaires sont plus élevées chez les élèves canadiens d'origine immigrée appartenant à des « minorités visibles » que chez leurs pairs non racisés (Krahn et Taylor, 2005). Anisef et Kilbride (2004) proposent ainsi le concept de résilience ethnique pour souligner cette capacité de surmonter les obstacles systémiques et structurels, et de réussir à s'adapter de façon exceptionnelle. Souvent victimes de marginalisation et de discrimination sur le marché de l'emploi, et vivant dans des conditions précaires et de pauvreté (Block et al., 2019 ; Branker, 2017), ces parents s'investissent beaucoup pour éviter que leurs enfants ne subissent le même sort plus tard et certains font beaucoup de sacrifices qui dépassent souvent leurs moyens pour scolariser leurs enfants jusqu'au postsecondaire (Anisef et al., 2011). Ces derniers sont alors poussés à travailler « deux fois plus fort » pour neutraliser les stéréotypes négatifs et réussir leurs études (McGee, 2020 ; McGee et al., 2019).

Enfin, la troisième piste explicative que nous pouvons avancer s'inspire de la théorie du choix rationnel soutenue par Boudon (1973). Elle renvoie à la détermination 
des élèves et de leurs parents. Même s'ils sont souvent relégués dans des programmes (classe spéciale) et des établissements de second ordre, les étudiants noirs d'origine immigrée de l'Afrique subsaharienne et des Caraïbes arrivent à s'en servir comme tremplins pour faire des choix plus prestigieux ; ils affirment fortement leur identité « ethnoraciale » comme moyen de faire leur chemin aux études postsecondaires et de configurer leur propre parcours (Collins et Magnan, 2018 ; Samuel et Burney, 2003 ; Sinacore et Lerner, 2013 ; Taylor et James, 2015 ; Wilson-Forsberg et al., 2018). Cette relégation, souvent fondée sur les stéréotypes et les préjugés raciaux, freine leurs ambitions et retarde leur parcours scolaire, mais ne brise pas pour autant le potentiel cognitif élevé dont une partie d'entre eux sont dotés. Au contraire, ils parviennent à l'activer pour contourner les obstacles.

\section{Conclusion}

Nos analyses sur l'accès au collège et à l'université, ainsi que l'obtention d'un diplôme collégial, révèlent que les jeunes noirs d'origine africaine et caribéenne sont moins susceptibles d'avoir fréquenté une bonne école (privée ou publique enrichie), mais plus susceptibles d'avoir vécu des expériences de difficultés scolaires, qu'ils aient accédé ou non au collège. Ces résultats témoignent aussi du fait que le parcours scolaire au secondaire et au primaire n'influe pas seulement sur l'accès aux études supérieures, mais également sur la persévérance aux études et les chances d'obtenir un diplôme. En conclusion, les parcours scolaires des jeunes noirs d'origine africaine et caribéenne sont caractérisés par de nombreux facteurs de risque liés à l'environnement social et scolaire. Ils présentent deux faces : celle de la résilience et celle du destin. Si la première témoigne la capacité indéniable de ces jeunes à mobiliser et à combiner un ensemble de facteurs de protection pour neutraliser les facteurs de risque, la deuxième marque les limites de la première qu'il ne faut pas non plus négliger.

Certes, le niveau élevé d'aspirations et d'engagement scolaires et la détermination à réussir constituent une stratégie, voire une condition essentielle pour contourner les obstacles liés au racisme et à la discrimination systémique, mais sont-ils suffisants pour accéder aux études postsecondaires, s'assurer un parcours régulier vers et aux études postsecondaires, obtenir le diplôme dans les mêmes conditions que les étudiants non 
racisés ? La réponse à cette question dépendrait des contextes sociétaux et des politiques publiques. Au Québec, elle est plutôt partagée. Il est indéniable que les pouvoirs publics n'ont cessé de mettre en place, depuis la réforme Parent (en 1964), des politiques pour favoriser la réussite et la persévérance de tous les élèves. Citons à cet égard les classes d'accueil pour les enfants immigrants nouvellement arrivés, les services de soutien aux élèves handicapés et en situation de difficultés d'adaptation scolaire et d'apprentissage, l'assouplissement des parcours vers les études postsecondaires, ainsi que la généralisation d'une formation commune (ou d'un tronc commun) jusqu'en 2e année du secondaire. En revanche, il y a également lieu de reconnaître que les mêmes pouvoirs publics ont progressivement balisé, voire soutenu de nouvelles pratiques institutionnelles favorisant l'exclusion sociale subtile en milieu scolaire, notamment par le biais de la stratification des établissements et la hiérarchisation des programmes d'enseignement (Kamanzi, 2018, 2019b). Cette exclusion touche en particulier les élèves issus des communautés ethniques dont font partie les familles immigrées de l'Afrique subsaharienne et caribéenne (Kamanzi et al., 2018). Dès lors, tout en soulignant ce que Herbaut (2019) appelle « l'avantage compensatoire » de la résilience, la présente étude amène à reconnaître ce que la même auteure qualifie de « désavantage cumulatif » du parcours scolaire au primaire et au secondaire caractérisant les élèves appartenant à des groupes racisés.

Sur le plan politique, les résultats apportent un éclairage sur les facteurs de production des inégalités sociales en éducation, mais aussi sur les pistes d'intervention. Plus précisément, l'accès relativement élevé des élèves québécois d'origine africaine et caribéenne aux études postsecondaires témoigne de leur engagement aux études, en dépit des obstacles inhérents à l'environnement social, lesquels expliquent en partie leur vulnérabilité. Dès lors, les pouvoirs publics sont appelés à mettre davantage l'accent sur l'amélioration des conditions d'apprentissage et sur le soutien à la réussite. Dans le contexte actuel, de telles mesures concernent non seulement les ressources éducatives (le temps, la qualité des activités scolaires et parascolaires et les ressources matérielles) pour compenser les lacunes inhérentes à l'origine sociale, mais viendraient également combler les effets des ségrégations socioscolaires auxquelles ils sont les plus exposés (Motti-Stefanidi et Masten, 2017). Cela dit, à long terme, la solution devrait plutôt être l'éradication du racisme sous-jacent et l'instauration d'une véritable justice sociale et scolaire où le recours aux mécanismes de résilience et de survie n'aurait que peu de place. 


\section{References}

Abada, T., Hou, F. et Ram, B. (2009). Ethnic differences in educational attainment among the children of Canadian immigrants. Canadian Journal of Sociology, 34(1), 1-28. https://doi.org/10.29173/cjs1651

Alba, R. et Foner, N. (2014). Comparing immigrant integration in North America and Western Europe: How much do the grand narratives tell us? International Migration Review, 48(1_Suppl.), 263-291. https://doi.org/10.1111/imre.12134

Allison, P. D. (2012). Logistic regression using the SAS: Theory and application (2e éd.). SAS Institute Inc.

Anaut, M. (2006). L'école peut-elle être facteur de résilience ? Empan, 63(3), 30-39. https://doi.org/10.3917/empa.063.0030

Anisef, P. et Kilbride, K. M. (2004). Introduction. Dans P. Anisef et K. M. Kilbride (dir.), Managing two worlds: The experiences and concerns of immigrant youth in Ontario (p. 1-36). Canadian Scholars' Press.

Anisef, P. S., Brown, R. S. et Sweet, R. (2011). Post-secondary pathway choices of immigrant and native-born youth in Toronto. Canadian Issues, (Winter), 42-48. https://acs-aec.ca/wp-content/uploads/2019/05/CITC-2011-Winter-Hiver-L-1.pdf

Bhamra, R., Dani, S. et Burnard, K. (2011). Resilience: The concept, a literature review and future directions. International Journal of Production Research, 49(18), 5375-5393. https://doi.org/10.1080/00207543.2011.563826

Bhopal, K. (2017). Addressing racial inequalities in higher education: Equity, inclusion and social justice. Ethnic and Racial Studies, 40(13), 2293-2299. https://doi.org/1 $\underline{0.1080 / 01419870.2017 .1344267}$

Block, S., Galabuzi, G.-E. et Tranjan, R. (2019). Canada's colour coded income inequality [rapport]. Ryerson University, Canadian Centre for Policy Alternatives. https://www.policyalternatives.ca/publications/reports/ canadas-colour-coded-income-inequality

Bloemraad, I. (2012, juillet). Understanding 'Canadian exceptionalism' in immigration and pluralism policy. Migration Policy Institute. http://www.migrationpolicy.org/ research/TCM-canadian-exceptionalism 
Boudon, R. (1973). L’inégalité des chances : La mobilité sociale dans les sociétés industrielles. Armand Colin.

Branker, R. R. (2017). Labour market discrimination: the lived experiences of Englishspeaking Caribbean immigrants in Toronto. Journal of International Migration \& Immigration, 18(1), 203-222. https://doi.org/10.1007/s12134-016-0469-x

Briggs, A. Q. (2019). "We had support from our brothers": a critical race counternarrative inquiry into second-generation Black Caribbean male youth responses to discriminatory work pathways. Journal of Education and Work, 32(4), 377-392. https://doi.org/10.1080/13639080.2019.1624696

Byrne, B. B. (2010). Structural equations modeling with AMOS: Basic concepts, applications, and programming (2e éd.). Routledge.

Cheng, L. et Yan, W. (2018). Immigrant student achievement and educational policy in Canada. Dans L. Volante, D. Klinger et O. Bilgili (dir.), Immigrant Student Achievement and Education Policy : Cross-cultural approaches (p. 137-153). Springer. https://doi.org/10.1007/978-3-319-74063-8

Collins, T. et Magnan, M. O. (2018). Post-secondary pathways among second-generation immigrant youth of Haitian origin in Quebec. Canadian Journal of Education, 41(2), 413-440. https://journals.sfu.ca/cje/index.php/cje-rce/article/view/2426

De Graauw, E. et Vermeulen, F. (2016). Cities and the politics of immigrant integration: a comparison of Berlin, Amsterdam, New York City, and San Francisco. Journal of Ethnic and Migration Studies, 42(6), 989-1012. https://doi.org/10.1080/13691 $\underline{83 X .2015 .1126089}$

Dei, G. J. S. (2013). Chapter one: Reframing critical anti-racist theory (CART) for contemporary times. Counterpoints, 445, 1-14. https://www.jstor.org/ $\underline{\text { stable/42982029 }}$

Delgado Bernal, D. (2002). Critical race theory, Latino critical theory, and critical raced-gendered epistemologies: Recognizing students of color as holders and creators of knowledge. Qualitative Inquiry, 8(1), 105-126. https://doi. org/10.1177/107780040200800107 
Estrada, A. X., Severt, J. B. et Jiménez-Rodríguez, M. (2016). Elaborating on the conceptual underpinnings of resilience. Industrial and Organizational Psychology, 9(2), 497-502. https://doi.org/10.1017/iop.2016.46

Felouzis, G., Liot, F. et Perroton, J. (2005). L'Apartheid scolaire : Enquête sur la ségrégation ethnique dans les collèges. Éditions du Seuil.

Finnie, R. et Mueller, R. E. (2008). The effects of family income, parental education and other background factors to post-secondary education in Canada. Measuring the effectiveness of student aid [MESA project research paper]. https://www.yorku.ca/ pathways/literature/Access/MESA_Finnie Mueller.pdf

Finnie, R. et Mueller, R. E. (2010). They came, they saw, they enrolled: Access to postsecondary education by the children of Canadian immigrants. Dans R. Finnie, M. Frenette, R. E. Mueller et A. Sweetman (dir.), Pursuing higher education in Canada : Economic, social, and policy dimensions (p. 192-216). Queen's University Press.

Gallopin, G. C. (2006). Linkages between vulnerability, resilience, and adaptive capacity. Global Environmental Change, 16(3), 293-303. https://doi.org/10.1016/j. gloenvcha.2006.02.004

Ghosh, R. (2004). Public education and multicultural policy in Canada: The special case of Quebec. International Review of Education, 50(5), 543-566. https://www.jstor. org/stable/4151617

Hadj Abdou, L. (2019). Immigrant integration: the governance of ethno-cultural differences. Comparative Migration Studies, 7(15), 2-8. https://doi.org/10.1186/ s40878-019-0124-8

Harles, J. C. (1997). Integration 'before’ assimilation: immigration, multiculturalism and the Canadian polity. Canadian Journal of Political Science, 30(4), 711-736. https://doi.org/10.1017/S0008423900016498

Harles, J. C. (2004). Immigrants integration in Canada and United States. American Review of Canadian Studies, 34(2), 223-258. https://doi. org/10.1080/02722010409481199

Henry, F. et Tator, C. (dir.). (2009). Racism in the Canadian university: Demanding social justice, inclusion, and equity. University of Toronto Press. 
Henry, F., Rees, T. et Tator, C. (2010). The colour of democracy: Racism in Canadian society (4e éd.). Nelson Education.

Herbaut, E. (2019). Les inégalités d'accès à l'enseignement supérieur français : Avantages cumulatif et compensatoire au cours de l'enseignement secondaire. Revue française de sociologie, 60(4), 535-566. https://doi.org/10.3917/ $\underline{\text { rfs.604.0535 }}$

Hochschild, J. L. et Cropper, P. (2010). Immigration regimes and schooling regimes: Which countries promote successful immigrant incorporation? Theory and Research in Education, 8(1), 21-61. https://scholar.harvard.edu/jlhochschild/publications/ immigration-regimes-and-schooling-regimes-which-countries-promote-successf

Hoyle, R. H. (2012). Handbook of structural equation modeling. Guilford Press.

Ichou, M. (2014). Who they were there: Immigrants' educational selectivity and their children's educational attainment. European Sociological Review, 30(6), 750-765. https://doi.org/10.1093/esr/jcu071

Institut de la Statistique du Québec [ISQ]. (2019). Panorama des régions du Québec. Gouvernement du Québec.

James, C. E. (2009). Masculinity, racialization, and schooling: The making of marginalized men. Dans W. Martino, M. Kehler et M. B. Weaver-Hightower (dir.), The problem with boys' education: beyond the backlash (p. 102-123). Routledge.

James, C. et Turner, T. (2017). Towards race equity in education: The schooling of Black students in the greater Toronto area. The Jean Augustine Chair in Education, Community and Diaspora. https://youthrex.com/wp-content/uploads/2019/05/ Towards-Race-Equity-in-Education-April-20172.pdf

Kamanzi, P. C. (2012). L'emprise des titres scolaires sur l'emploi chez les membres de minorités visibles : comparaison entre le Québec et l'Ontario. Recherches sociographiques, 53(2), 313-334.

Kamanzi, P. C. (2018). L'évolution récente de la démocratisation de l'éducation au Québec : quand l'inclusion fait place à la stratification. Cahiers de recherche sociologique, (64), 203-225. https://doi.org/10.7202/1064726ar 
Kamanzi, P. C. (2019a). Les parcours scolaires des jeunes canadiens d'origine immigrante aux études supérieures : qui y accède et comment ? Educaçao \& Sociedade, 40, 1-19. https://doi.org/10.1590/es0101-73302019181525

Kamanzi, P. C. (2019b). School market in Quebec and the reproduction of social inequalities in higher education. Social Inclusion, 7(1), 18-27. https://doi. org/10.17645/si.v7i1.1613

Kamanzi, P. C. et Collins, T. (2018). The postsecondary education pathways of Canadian immigrants: Who goes and how do they get there. International Journal of Social Science Studies, 6(2), 58-68. https://doi.org/10.11114/ijsss.v6i2.2866

Kamanzi, P. C. et Murdoch, J. (2011). L'accès à un diplôme universitaire chez les immigrants. Dans F. Kanouté et G. Lafortune (dir.), Familles québécoises d'origine immigrante : Les dynamiques de l'établissement (p. 145-158). Presses de l'Université de Montréal.

Kamanzi, P. C., Bastien, N., Doray, P. et Magnan, M.-O. (2016). Immigration et cheminements scolaires aux études supérieures au Canada : qui y va et quand? Une analyse longitudinale à partir du modèle de Cox. Revue canadienne d'enseignement supérieur, 46(2), 225-248. https://doi.org/10.47678/cjhe. v46i2.184865

Kamanzi, P. C., Magnan, M.-O., Pilote, A. et Doray, P. (2018). Immigration et morphologie des parcours scolaires dans l'enseignement supérieur au Canada : le cas de la province de Québec. Revue Européenne des Migrations Internationales, 34(2/3), 253-277. https://doi.org/10.4000/remi.11280

Kamanzi, P. C., Pilote, A., Uzenat, M. et Gris, S. (2017). La démocratisation des études supérieures à l'aune de la différenciation et de l'individualisation des parcours scolaires au Québec. L'orientation scolaire et professionnelle, 46(4), 571-594. https://doi.org/10.4000/osp.5527

Kanouté, F. et Lafortune, G. (2010). Les familles immigrantes : mobilisation autour du projet scolaire des enfants. Nos diverses cités, (7), 143-150.

Kline, R. B. (2011). Principles and practice of structural equation modeling (3e éd.). Guilford Press. 
Koopmans, R. (2013). Multiculturalism and immigration: A contested field in crossnational comparison. Annual Review of Sociology, 39, 147-169. https://doi. org/10.1146/annurev-soc-071312-145630

Krahn, H. et Taylor, A. (2005). Resilient teenagers: Explaining the high educational aspirations of visible minority youth in Canada. Journal of International Migration and Integration, 6(3-4), 405-434. https://doi.org/10.1007/ s12134-005-1020-7

Ladson-Billings, G. (1998). Just what is critical race theory and what's it doing in a nice field like education? International Journal of Qualitative Studies in Education, 11(1), 7-24. https://doi.org/10.1080/095183998236863

Lafortune, G. (2012). Rapport à l'école et aux savoirs scolaires de jeunes d'origine haïtienne en contexte scolaire défavorisé à Montréal [Thèse de doctorat]. Université de Montréal. http://hdl.handle.net/1866/8479

Levels, M., Dronkers, J. et Kraaykamp, G. (2008). Immigrant children's educational achievement in western countries: origin, destination, and community effects on mathematical performance. American Sociological Review, 73(5), 835-853. https://doi.org/10.1177/000312240807300507

Livingstone, A.-M. et Weinfeld, M. (2017). Black students and high school completion in Quebec and Ontario : A multivariate analysis. Canadian Review of Sociology, 54(2), 174-197. https://doi.org/10.1111/cars.12144

Luthans, F., Avey, J. B., Avolio, B. J., Norman, S. M. et Combs, G. M. (2006). Psychological capital development: Toward a micro-intervention. Journal of Organizational Behavior, 27(3), 387-393. https://doi.org/10.1002/job.373

Magnan, M.-O., Pilote, A., Collins, T. et Kamanzi, P. C. (2019). Discours de jeunes issus de groupes minoritaires sur les inégalités scolaires au Québec. Diversité urbaine, 19, 93-114. https://doi.org/10.7202/1065122ar

Magnan, M.-O., Pilote, A., Grenier, V. et Darchinian, F. (2017). Jeunes issus de l'immigration et choix d'orientation au postsecondaire à Montréal. Revue canadienne d'enseignement supérieur, 47(3), 34-53. https://doi. org/10.7202/1043237ar 
Magnan, M.-O., Pilote, A., Grenier, V. et Kamanzi, P. C. (2017). Le rôle de la socialisation familiale dans les choix d'études supérieures des jeunes issus de l'immigration. Revue Jeunes et Société, 2(1), 30-58. http://rjs.inrs.ca/index.php/ rjs/article/view/87-48

Manciaux, M. (2001). La résilience. Un regard qui fait vivre. Études, 395(10), 321-330. https://doi.org/10.3917/etu.954.0321

Mc Andrew, M., Ledent, J., Murdoch, J., Ait-Said, R. et Balde, A. (2013). Le profil et le cheminement scolaire des jeunes québécois issus de l'immigration au secondaire : un portrait statistique. Cahiers québécois de démographie, 42(1), 31-55. https:// doi.org/10.7202/1017097ar

McGee, E. O. (2020). Interrogating structural racism in STEM higher education. Educational Researcher, 49(9), 633-644. https://doi. org/10.3102/0013189X20972718

McGee, E. O., Griffith, D. M. et Houston, S. (2019). I know I have to work twice as hard and hope that makes me good enough: Exploring the stress and strain of Black doctoral students in engineering and computing. Teachers College Record, 121(4), 1-38. http://www.tcrecord.org/Content.asp?ContentId=22610

Mickelson, R. A. (2003). When are racial disparities in education the result of racial discrimination? A social science perspective. Teachers College Record, 105(6), 1052-1086. https://doi.org/10.1111/1467-9620.00277

Motti-Stefanidi, F. (2018). Resilience among immigrant youth: The role of culture, development and acculturation. Developmental Review, 50(Part A), 99-109. https://doi.org/10.1016/j.dr.2018.04.002

Motti-Stefanidi, F. et Masten, A. S. (2017). A resilience perspective on immigrant youth adaptation and development. Dans N. J. Cabrera et B. Leyendecker (dir.), Handbook on positive development of minority children and youth (p. 19-34). Springer. https://doi.org/10.1007/978-3-319-43645-6_2

Organisation de coopération et de développement économiques et Union européenne [OCDE/UE]. (2018). Settling in 2018: Indicators of immigrant integration. https://doi.org/10.1787/9789264307216-en 
Pettit, P. (2007) Resilience as the explanandum of social theory. Dans I. Shapiro et S. Bedi (dir.), Studying the unexpected, the accidental, and the unforeseen (p. 79-96). New York University Press.

Picot, G. (2012). Immigrant status and secondary school performance as determinants of post-secondary participation: A Comparison of Canada and Switzerland [Document de travail no 77]. OCDE. http://dx.doi.org/10.1787/5k9909jhz4wl-en

Potochnick, S. (2014). The academic adaptation of children of immigrants in new and established settlement states: The role of family, schools, and neighborhoods. Population Research and Policy Review, 33(3), 335-364. https://doi.org/10.1007/ $\underline{\text { s11113-013-9319-0 }}$

Potvin, M. et Leclercq, J.-B. (2014). Facteurs affectant la trajectoire scolaire des jeunes de 16-24 ans issus de l'immigration en formation générale des adultes. Revue des sciences de l'éducation, 40(2), 309-349. https://doi.org/10.7202/1028423ar

Robson, K., Anisef, P., Brown, R. S. et George, R. (2018). Underrepresented students and the transition to postsecondary education: Comparing two Toronto cohorts. Canadian Journal of Higher Education, 48(1), 39-59. https://doi. org/10.7202/1050841ar

Rogers-Sirin, L., Ryce, P. et Sirin, S. R. (2014). Acculturation, acculturative stress, and cultural mismatch and their influences on immigrant children and adolescents' well-being. Dans R. Dimitrova, M. Bender et F. van de Vijver (dir.), Global perspectives on well-being in immigrant families (p. 11-30). Springer.

Rothon, C., Heat, A. et Lessard-Philips, L. (2009). The educational attainments of the "second generation": A comparative study of Britain, Canada, and the United States. Teachers College Record, 111(6), 1404-1443.

Safi, M. (2008). The immigrant integration process in France: Inequalities and segmentation. Revue française de sociologie, 49(5), 3-44. https://doi.org/10.3917/ $\underline{\text { rfs.495.0003 }}$

Sala Pala, V. (2007). La politique du logement social est-elle raciste ? L'exemple marseillais. Faire Savoirs, (6), 25-36. http://faire-savoirs.mmsh.univ-aix.fr/Pdf/ FS-6-2007-092.pdf 
Samuel, E. et Burney, S. (2003). Racism, eh? Interactions of South Asian students with mainstream faculty in a predominantly white Canadian university. The Canadian Journal of Higher Education, 33(2), 81-114. https://doi.org/10.47678/cjhe. $\underline{\mathrm{v} 33 \mathrm{i} 2.183433}$

Silver, H. (2015). The contexts of social inclusion [Document de travail no 144]. Department of Economic and Social Affairs. www.un.org/esa/desa/papers/2015/ wp144_2015.pdf

Simich, L., Beiser, M., Stewart, M. et Mwakarimba, E. (2005). Providing social support for immigration and refugees in Canada: Challenges and directions. Journal of Immigrant and Minority Health, 7(4), 259-268. https://doi.org/10.1007/ s10903-005-5123-1

Sinacore, A. L. et Lerner, A. (2013). The cultural and educational transitioning of first generation immigrant undergraduate students in Quebec, Canada. International Journal for Educational and Vocational Guidance, 13(1), 67-85. https://doi. org/10.1007/s10775-013-9238-y

Sleeter, C. E. (2012). Critical race theory and education. Dans J. A. Banks (dir.), Encyclopedia of diversity in education, Volume 1 (p. 491-495). SAGE.

Söhn, J. et Özcan, V. (2006). The educational attainment of Turkish migrants in Germany. Turkish Studies, 7(1), 101-124. https://doi.org/10.1080/14683840500520626

Solorzano, D. G. (1998). Critical race theory, race and gender microaggressions, and the experience of Chicana and Chicano scholars. International Journal of Qualitative Studies in Education, 11(1), 121-136. https://doi.org/10.1080/095183998236926

Sweet, R., Anisef, P., Brown, R., Walters, D. et Phythian, K. (2010). Post-High School Pathways of Immigrant Youth [Rapport]. Higher Education Quality Council of Ontario. https://heqco.ca/pub/post-high-school-pathways-of-immigrant-youth/

Taylor, L. et James, C. E. (2015). Living up to expectations: 2nd and 1.5-generation immigrant students' pursuit of university education. Dans G. Man et R. Cohen (dir.), Engendering transnational voices : Studies in family, work, and identity (chap. 9). Wilfrid Laurier University Press. 
CAPSUle SPÉCIALE : LA RÉUSSITE ÉDUCATIVE DES AFRO-CANADIENS

Thiessen, V. (2009). The pursuit of postsecondary education: A comparison of First Nations, African, Asian, and European Canadian Youth. Canadian Review of Sociology, 46(1), 5-37. https://doi.org/10.1111/j.1755-618X.2009.01201.x

Walker, C., Gleaves, A. et Grey, J. (2006). Can students within higher education learn to be resilient and, educationally speaking, does it matter? Educational Studies, 32(3), 251-264. https://doi.org/10.1080/03055690600631184

Wilson-Forsberg, S., Masakure, O., Shizha, E., Lafrenière, G. et Mfoafo-M'Carthy, M. (2018). Disrupting an imposed racial identity or performing the model minority? The pursuit of postsecondary education by young African immigrant men in Southern Ontario, Canada. Race Ethnicity and Education, 23(5), 693-711. https:// doi.org/10.1080/13613324.2018.1497965 